\title{
Elaboração de salsicha de peixe pargo Pagrus pagrus de tamanho reduzido proveniente da pesca de arrasto com baixo valor comercial
}

\author{
Development of fish sausage using low commercial value \\ red porgy Pagrus pagrus
}

\author{
Juliana de Lima Brandão Guimarães, ${ }^{* *}$ Flávia Aline Andrade Calixto, ${ }^{* *}$ Luiz Antônio de Moura Keller, ${ }^{* * *}$ \\ Juliana Tomaz Pacheco Latini, ${ }^{* * * *}$ Angela Aparecida Lemos Furtado, ${ }^{* * * *}$ Eliana de Fátima Marques de Mesquita ${ }^{* * * * * *}$
}

\begin{abstract}
Resumo
O objetivo deste trabalho foi desenvolver salsicha utilizando o pargo Pagrus pagrus de baixo valor comercial capturado na modalidade de pesca de arrasto e classificado como na categoria "mistura" por ter tamanho reduzido para o mercado varejista e realizar análises físico-químicas, microbiológicas, toxicológicas e sensoriais. A salsicha foi elaborada na planta piloto da Embrapa Agroindústria de Alimentos utilizando $50 \%$ de surimi e $50 \%$ de filé. As análises físico-químicas, microbiológicas e toxicológicas foram realizadas com métodos oficiais. O teste de aceitação foi realizado com 87 provadores que avaliaram o produto utilizando escala hedônica de sete pontos e também arguidos quanto à intenção de compra do produto. O produto foi considerado aceito quando $70 \%$ dos provadores atribuíram nota $\geq 4$. Os resultados da composição centesimal foram: umidade $71,22 \%$, proteínas $15,34 \%$, lipídios totais $5,55 \%$, carboidratos $6,11 \%$, cinzas $1,78 \%$ e o valor energético $135,75 \mathrm{kcal}$. As avaliações microbiológicas e toxicológicas mostraram que os produtos apresentaram qualidade satisfatória conforme a legislação. A salsicha foi aceita por $82,7 \%$ dos provadores e o aspecto global do produto atingiu média de $4,93( \pm 1,69)$. Em relação à intenção de compra foi atribuída a maior porcentagem para talvez sim/talvez não (32\%), seguido de provavelmente compraria $(24 \%)$, e $28,1 \%$ informaram que provavelmente ou decididamente não comprariam. Com base nos resultados obtidos, conclui-se que o peixe de baixo valor agregado da categoria "mistura" resultou em produto embutido viável para o uso na tecnologia do pescado.
\end{abstract}

Palavras-chave: categoria mistura da pesca de arrasto, derivados de pescado, análises bacteriológicas, análises físico-químicas, aceitação.

\begin{abstract}
The aim of this work was to develop sausage using the low commercial value red porgy (Pagrus pagrus) caught in the "fish mix" category in the trawl fishery and to perform physicochemical, microbiological, toxicological and sensorial analysis. The sausage was prepared using $50 \%$ of surimi and $50 \%$ of fillet in Embrapa Food Agroindustry Pilot Plant. The physicochemical, microbiological, toxicological analyses were performed with official methods. The sensorial analyses were performed with 87 testers who evaluated the product using a hedonic scale of seven (7) points and were also accused of buying the product. The product was considered accepted when $70 \%$ of the tasters assigned a score $\geq 4$. One sausage formulation was tested using $50 \%$ surimi. The results of the centesimal composition were: moisture $71.22 \%$, proteins $15.34 \%$, total lipids $5.55 \%$, carbohydrates $6.11 \%$, ash $1.78 \%$ and energy value $135.75 \mathrm{kcal}$. The parameters of microbiological and toxicological are within the current legislation for breaded products. The sausage was accepted obtaining $82.7 \%$ and the overall impression of the product reached a mean of $4.93( \pm 1.69)$. Regarding intention to purchase for sausage, the highest percentage was attributed to maybe / perhaps not (32\%), followed by likely to buy $(24 \%)$, and $28.1 \%$ reported that they probably or decidedly would not buy. Based on the results obtained, it was concluded that the low commercial value fish of the "fish mix" category resulted in a viable product available for use in fish technology.
\end{abstract}

Keywords: bycatch, byproducts, bacteriological analyzes, physicochemical analyzes, acceptability.

${ }^{*}$ Recebido em 18 de maio de 2020 e aceito em 1 de outubro de 2020.

**Fundação Instituto de Pesca do Estado do Rio de Janeiro, FIPERJ, DPP, Niterói, Rio de Janeiro, Brasil. *E-mail: julianafiperj@gmail.com,

***Universidade Federal Fluminense, UFF, Faculdade de Veterinária, MZO, Niterói, RJ, Brasil.

****Universidade Federal do Rio de Janeiro, UFRJ, Campus Macaé, Macaé, RJ, Brasil.

*****Embrapa Agroindústria de Alimentos, RJ, Brasil.

******Universidade Federal Fluminense, UFF, Faculdade de Veterinária, MTA, Niterói, RJ, Brasil. 


\section{Introdução}

O pargo Pagrus pagrus (Linnaeus, 1758) é um peixe marinho, demersal com ampla distribuição geográfica, incluindo Oceano Atlântico e Mar Mediterrâneo. Tem uma importância comercial bastante significativa, sendo comercializado entre os estados do Espírito Santo e Rio Grande do Sul servindo ao mercado interno e à exportação (Haimovici et al., 1994). A modalidade de pesca mais utilizada para a captura do pargo são os covos (FIPERJ, 2013), porém, é também capturado com frequência na pesca de arrasto como espécie não-alvo, e, por apresentar tamanho reduzido, é incluído na categoria "mistura" e comercializado com baixo valor (Quirino-Duarte et al., 2009).

Devido à baixa seletividade e ao reduzido tamanho das malhas das redes tradicionais o arrasto tornou-se responsável pelas maiores capturas de fauna-acompanhante (Vianna et al., 2003). Somente para a pescaria de camarão, estima-se uma produção anual de 1,3 milhão de toneladas e aproximadamente 7,3 milhões de toneladas de captura descartada (Kelleher, 2005).

O aproveitamento dos peixes da categoria "mistura" no desenvolvimento de novos produtos pode ser alternativa viável para fornecer proteína de excelente qualidade e minimizar impactos (Pires et al., 2014). A utilização de espécies de peixes com baixa expressão econômica vem sendo tema de pesquisa no Brasil. Peixes de baixo valor comercial têm sido utilizados como matéria-prima na produção de carne mecanicamente separada de pescado (CMSP), surimi e embutidos (Tenuta-Filho e Jesus, 2003; Lanfer-Marquez e Mira, 2005). Contudo, para elaborar derivados de pescado, independente do processamento do pescado ser realizado em uma indústria ou de forma artesanal, é exigido um controle de qualidade tanto quanto à sanidade do pescado e à utilização de sistemas que garantam a inocuidade do produto. (Gonçalves, 2011).

Segundo a legislação brasileira, a carne mecanicamente separada de pescado é o produto congelado obtido de pescado, envolvendo o descabeçamento, a evisceração, a limpeza deste e a separação mecânica da carne das demais estruturas inerentes à espécie, como espinhas, ossos e pele (BRASIL, 2017).

O surimi é obtido a partir da CMSP de resíduos do processamento do pescado, submetida a lavagens sucessivas, retirada do excesso de água, adição de crioprotetores e congelamento para sua preservação (Lee, 1984). O surimi apresenta características, tais como ausência de odor de peixe, alto teor protéico e baixo teor de lipídeo podendo ser acrescentado a uma variedade de alimentos como os embutidos (Alfaro et al., 2004).

O objetivo deste trabalho foi desenvolver salsicha utilizando o pargo Pagrus pagrus de baixo valor comercial capturado na modalidade de pesca de arrasto e classificado como na categoria "mistura" por ter tamanho reduzido para o mercado varejista e realizar análises físico-químicas, microbiológicas, toxicológicas e sensoriais.

\section{Material e métodos}

Para a elaboração da salsicha, realizou-se a coleta de 30 quilogramas de pargo para processamento. Os peixes foram coletados no momento do desembarque em cais no município de São Gonçalo (RJ) e transportados em caixas isotérmicas com gelo na proporção 1:1 para o laboratório da Fundação Instituto de Pesca do Estado do Rio de Janeiro (FIPERJ) que fica a 500m de distância. No laboratório, os peixes foram lavados em água potável provenientes da estação de tratamento local, eviscerados, descabeçados e filetados. Os filés e o resíduo de filetagem foram embalados separadamente em sacos plásticos e armazenados em freezer $\mathrm{a}-18^{\circ} \mathrm{C}$.

Os filés e resíduo de filetagem congelados foram transportados em caixas isotérmicas para o Laboratório de Processamento de Pescado da Embrapa Agroindústria de Alimentos (CTAA/ EMBRAPA). O processamento foi realizado conforme fluxograma (Figura 1). Após este processo, foram retiradas amostras para análises.

Para o desenvolvimento da Carne Mecanicamente Separada de Pescado (CMSP), o resíduo de filetagem (esqueleto ósseo e pele) foi processado na máquina de CMS para pescado marca Mec Pescado, Brasil. Após este processo, a CMSP foi pesada e foram retiradas amostras para análise.

O surimi foi obtido com um ciclo de lavagem com água a $5^{\circ} \mathrm{C}$ na proporção 3:1 (água: carne). A mistura água e CMSP foi agitada por cinco minutos e depois foi deixada em repouso por dois minutos. Foi retirado o excesso de água manualmente com tela de 100 micrômetros e depois com bomba à vácuo por cinco minutos. Ao final do processamento, foram adicionados crioprotetores: $2 \%$ de cloreto de sódio e $1 \%$ de sacarose. Após esta etapa, o surimi foi pesado, amostras coletadas e congeladas $\left(-18^{\circ} \mathrm{C}\right)$.

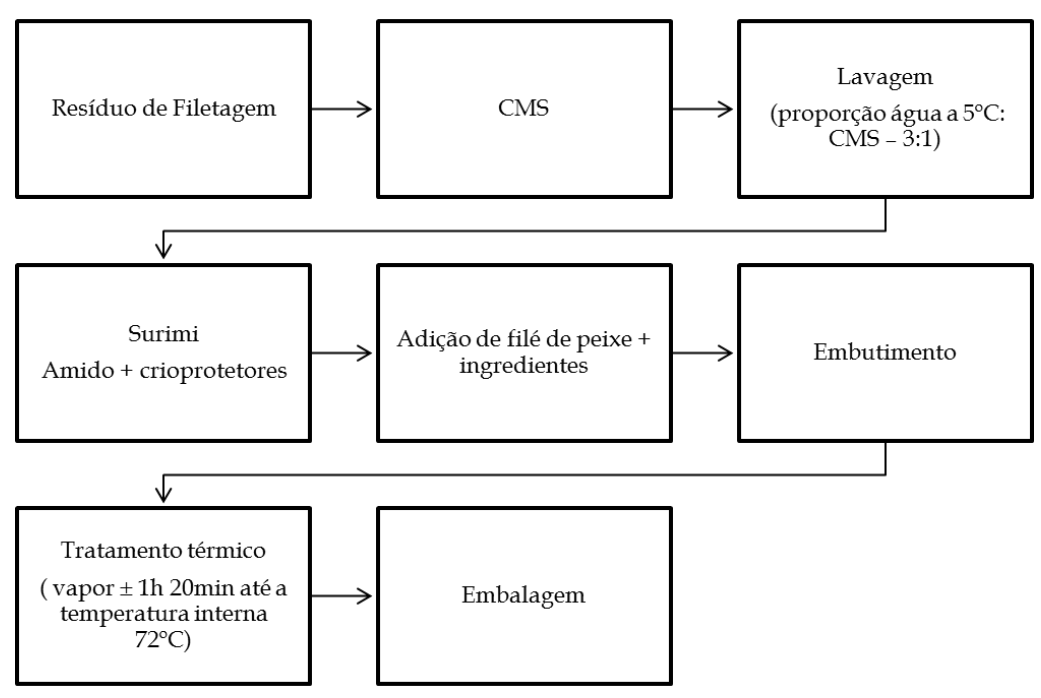

Figura 1: Fluxograma do processo de elaboração da salsicha de peixe 
Foi elaborada uma formulação de salsicha (50\% surimi $+35 \%$ filé $+15 \%$ ingredientes: proteína de soja, sal refinado, sal de cura, lactato de sódio, polifosfato de sódio, cebola desidratada, eritorbato, alho desidratado, pimenta branca, salsa desidratada, essência de limão). O surimi, filé e ingredientes foram misturados em "cutter" (5 minutos) até se obter uma emulsão. Após o processo de emulsificação, a massa foi retirada do "cutter" e levada à embutidora manual utilizando tripas de colágeno. Em seguida, foram amarradas a cada $10 \mathrm{~cm}$ de comprimento usando barbante de algodão.

O tratamento térmico foi realizado com vapor direto por aproximadamente 1 hora e 20 minutos, até a temperatura interna atingir $72^{\circ} \mathrm{C}$. Após o cozimento, as salsichas foram resfriadas por aspersão de água até temperatura interna de $40^{\circ} \mathrm{C}$. Foram embaladas a vácuo e estocadas sob refrigeração $\left(-18^{\circ} \mathrm{C}\right)$ até o momento das análises.

As análises físico-químicas realizadas para as amostras foram: matéria pré-seca a $105^{\circ} \mathrm{C}$ (umidade); cinzas; proteína bruta (MicroKjedahl); extrato etéreo (método de Soxlet) para a determinação da composição centesimal e assegurar credibilidade nos dados das análises quantitativas, os métodos analíticos serão validados, seguindo metodologia descrita no manual de métodos oficiais de análises da AOAC (2007). Todas as amostras dos ensaios foram avaliadas em triplicata nos laboratórios do Centro Estadual de Controle de Pesquisa em Qualidade de Alimentos (CEPQA), localizado na Empresa de Pesquisa Agropecuária do Estado do Rio de Janeiro (PESAGRO-RJ). Os carboidratos (extrativo não nitrogenado) foram calculados pela fração "NIFEXT" ("Nitrogen Free Extract"), tendo como parâmetro a diferença entre $100 \%$ das demais frações da composição centesimal (Oliveira et al., 1999). Com base nos valores de carboidratos, proteínas e lipídeos, foi calculado o valor energético total (VET) dos alimentos, sabendose que os carboidratos e proteínas fornecem $4 \mathrm{kcal} / \mathrm{g}$ de energia e os lipídeos $9 \mathrm{kcal} / \mathrm{g}$ (FAO, 2005).

As análises microbiológicas realizadas foram: pesquisa de Salmonella spp. e contagens de coliformes a $35^{\circ} \mathrm{C}$, de coliformes termotolerantes e de Staphylococcus coagulase positiva. Todas as análises foram realizadas segundo metodologia da Instrução Normativa n 62 de 26 de agosto de 2003 do Ministério da Agricultura (Brasil, 2003) no Laboratório de Microbiologia da Embrapa Agroindústria de Alimentos.

Para avaliação toxicológica foi pesquisada a presença de gás sulfídrico $\left(\mathrm{H}_{2} \mathrm{~S}\right)$, segundo técnica descrita por LANARA (Brasil, 1981), e o teor de histaminas determinado conforme metodologia espectrofotofluométrica descrita por Glória \& Soares (1993). Também nos laboratórios do Centro Estadual de Controle de Pesquisa em Qualidade de Alimentos (CEPQA), localizado na Empresa de Pesquisa Agropecuária do Estado do Rio de Janeiro (PESAGRO-RJ).

O teste de aceitação foi realizado no Laboratório de Análise Sensorial da Faculdade de Veterinária, UFF e foram aplicados com o projeto "Aceitabilidade de produtos desenvolvidos com peixes da categoria "mistura" provenientes da pescaria de arrasto de camarão" aprovado pelo Comitê de Ética em Pesquisa da Faculdade de Medicina / Hospital Universitário Antônio Pedro - CEP CMM/HUAP no 1.934.771 - CAAE n. 63083716.5.0000.5243.

As salsichas foram aquecidas em água fervente por dois minutos, cortadas em pedaços de $2 \mathrm{~cm}$ de comprimento e servidas juntamente com água natural e biscoito de água. Todos os voluntários receberam e assinaram previamente um termo de consentimento livre e esclarecido, garantindo a sua aceitação para participar do teste. $O$ teste foi realizado com provadores não treinados, estudantes e funcionários da UFF, de ambos os sexos. O teste de aceitação foi realizado segundo metodologia de Dutcosky (2011), em cabines individuais, sob a luz branca e em temperatura ambiente.

O teste sensorial foi realizado com 87 provadores, que avaliaram o produto utilizando escala hedônica de sete pontos para os atributos odor, cor, textura e sabor e por fim, o aspecto global do produto. As possíveis observações poderiam ser apontadas pelos provadores na própria ficha. Por fim, foi indagado sobre a intenção de compra do produto dividido em escala de cinco pontos: decididamente compraria, provavelmente compraria, talvez sim/ talvez não, provavelmente eu não compraria, decididamente eu não compraria. Nos resultados obtidos pelo método da escala hedônica, as amostras foram aceitas se $70 \%$ dos provadores atribuíram nota $\geq 4$.

\section{Resultados e Discussão}

A Tabela 1 expressa os resultados da composição centesimal do produto de pargo.

$\mathrm{Na}$ Tabela 2 encontram-se os resultados das análises microbiológicas da salsicha de pargo de acordo com a legislação.
Tabela 1: Resultados médios da composição centesimal dos produtos de pargo (Pagrus pagrus)

\begin{tabular}{ccccccc}
\hline Produto & $\begin{array}{c}\text { Umidade } \\
(\%)^{*}\end{array}$ & $\begin{array}{c}\text { Proteína } \\
(\%)^{*}\end{array}$ & $\begin{array}{c}\text { Lipídeos } \\
(\%)^{*}\end{array}$ & $\begin{array}{c}\text { Carboidrato } \\
(\%)^{*}\end{array}$ & $\begin{array}{c}\text { Cinzas } \\
(\%)^{*}\end{array}$ & $\begin{array}{c}\text { Valor energético } \\
(\mathrm{kcal})^{* *}\end{array}$ \\
\hline Salsicha & 71,22 & 15,34 & 5,55 & 6,11 & 1,78 & 135,75
\end{tabular}

Legenda: * \% = percentual; gramas por 100 gramas de alimento. ${ }^{* *} \mathrm{kcal}=$ quilocaloria

Tabela 2: Resultados das análises microbiológicas da salsicha de pargo (Pagrus pagrus)

\begin{tabular}{ccccc}
\hline Produto & Salmonella sp. & Coliforme a $35^{\circ} \mathrm{C}$ & $\begin{array}{c}\text { Coliforme } \\
\text { termotolerante }\end{array}$ & $\begin{array}{c}\text { Estafilococos } \\
\text { coagulase positiva }\end{array}$ \\
\hline Salsicha & AUSENTE $/ 25 \mathrm{~g}^{*}$ & $<10 \mathrm{UFC} / \mathrm{g}^{* *}$ & $<10 \mathrm{UFC} / \mathrm{g}^{* *}$ & $<10^{2} \mathrm{UFC} / \mathrm{g}^{* *}$ \\
\hline
\end{tabular}

Legenda: * Ausente/25g = ausente em vinte e cinco gramas de alimento. ${ }^{*} \mathrm{UFC} / \mathrm{g}=$ unidade formadora de colônia por grama de alimento

Para as análises toxicológicas, foram avaliadas primeiramente a presença de compostos a base de enxofre em todas as amostras de salsichas de pargo, não sendo detectados níveis 
destes nas amostras. Também avaliados os níveis de histamina, onde foram estabelecidos limite de detecção (LOD) e o limite de quantificação (LOQ), determinados em 1,85 $\mathrm{mg} \mathrm{kg}^{-1}$ e 7,88 mg $\mathrm{kg}^{-1}$, respectivamente para a metodologia estabelecida, sendo que nenhuma das amostras apresentaram níveis menores do que LOD.

Para a análise sensorial, o total de provadores foi de 87 pessoas, sendo que $64,4 \%$ eram do sexo feminino e $35,6 \%$ do sexo masculino, com idade entre 20 a 65 anos. O produto foi aceito com $82,7 \%$ para o aspecto global do produto atingindo média de $4,82( \pm 1,67)$. Os resultados da aceitabilidade por atributo foram: Odor 85,05\%; Cor 66,66\%; Sabor 85,05\%; Textura 70,1\%.

O teste de Friedmann, ao nível de significância $\alpha=0,05$ (5\%), indica evidência de diferença estatisticamente significativa entre os escores dos quatro atributos $\left(\chi^{2}=21,545\right.$; graus de liberdade $=3 ; p<0,0001)$. A identificação das diferenças entre os atributos (comparações múltiplas) é realizada pelo teste exato do sinal, com significância corrigida para $\alpha^{*}=0,008$ pelo critério de Bonferroni. Os achados estão expressos na Tabela 3. Observa-se que há diferença estatística entre a categoria odor das categorias cor e textura. As categorias que apresentaram os resultados mais favoráveis foram odor e sabor e não diferiram estatisticamente entre si.

Tabela 3: Diferença estatisticamente significativa entre as quatro categorias (odor, cor, textura e sabor) analisadas sensorialmente pelo teste de Friedmann

\begin{tabular}{llll}
\hline \multicolumn{1}{c}{ Atributos } & \multicolumn{1}{c}{ Cor } & \multicolumn{1}{c}{ Textura } & \multicolumn{1}{c}{ Sabor } \\
\hline \multirow{4}{*}{ Odor } & $\mathrm{z}=-5.037$ & $\mathrm{z}=-3.887$ & $\mathrm{z}=-1.991$ \\
& $\mathrm{p}$-value $=.000$ & $\mathrm{p}$-value $=.000$ & $\mathrm{p}$-value $=.046$ \\
& SIM & SIM & NÃO \\
\hline \multirow{3}{*}{ Cor } & $\mathrm{z}=-2.163$ & $\mathrm{z}=-3.406$ \\
& $\mathrm{p}$-value $=.030$ & $\mathrm{p}$-value $=.000$ \\
& NÃO & SIM \\
Textura & & $\mathrm{z}=-2.380$ \\
& & $\mathrm{p}$-value $=.017$ \\
& & NÃO \\
\hline
\end{tabular}

O "SIM" indica diferença estatisticamente significativa $(p<.008)$ entre o escore médio do atributo indicado na respectiva linha e o escore médio do atributo indicado na respectiva coluna; o NÃO indica inexistência de diferença estatisticamente significativa ( $p>$.008) entre os escores dos atributos indicados nas respectivas linhas e colunas

Em relação à intenção de compra foi atribuída a maior porcentagem para talvez sim/talvez não $(32,1 \%)$, seguido de provavelmente compraria $(24,1 \%)$ e decididamente compraria $(14,9 \%)$, e $28,6 \%$ de respostas negativas ao produto.

O teor de umidade obtido na salsicha de pargo encontrou- se dentro da faixa do resultado obtido por Gonçalves et al. (2009) $(70,74 \%)$ em estudo com desenvolvimento de salsicha de piramutaba a partir do surimi. Valor de $82,43 \%$, superior ao trabalho atual, foi obtido por Marques et al. (2012) em estudo que avaliou a qualidade da linguiça de tilápia do Nilo a partir do filé.
A legislação brasileira afirma que as salsichas comuns, elaboradas com carne bovina, suína ou de aves, devem possuir os seguintes valores de composição química: umidade máxima de $65 \%$, proteína mínima de $12 \%$ e lipídeo máximo de $30 \%$ (Brasil, 2000). Portanto, de acordo com a legislação, a salsicha de pargo atendeu as exigências em proteína, porém apresentaram valores mais altos de umidade $(71,55 \%)$ que o preconizado pela legislação. Estes valores, acima do recomendado são inerentes à composição da carne de pescado utilizada e também pela ausência de gorduras de outras fontes, o que resultou em produto com alto teor de umidade e com teores de lipídeos bem abaixo do limite máximo preconizado pela legislação. Quanto aos níveis proteicos, o resultado encontrado por Gonçalves et al. (2009) foi de $12,6 \%$ em pesquisa com salsicha de piramutaba (Brachyplatystoma vaillantii) estando na mesma faixa de resultados de proteína do presente trabalho $(15,34 \%)$. Em outra com o desenvolvimento de linguiça de tilápia, Marques et al. (2012), encontraram valores proteicos de $10,48 \%$ estando abaixo dos valores de proteína para a salsicha de pargo no trabalho atual. Com relação aos valores obtidos para lipídios, o presente estudo encontrou resultado de $5,55 \%$ estando acima do teor de $0,06 \%$ encontrado por Peixoto et al. (2000) quando utilizou a espécie pescada-gó (Macrodon ancylodon) para o desenvolvimento de um moldado sabor camarão. Por outro lado, ainda na pesquisa de Peixoto et al. (2000) quando comparamos os teores de cinzas encontramos valores de $1,78 \%$ para a salsicha de pargo e de $2,59 \%$ para o moldado de camarão, possivelmente explicado pela presença do camarão na formulação.

A resolução RDC $n^{\circ} 12$, da Agência Nacional de Vigilância Sanitária - ANVISA (Brasil, 2001) estabelece que a tolerância máxima permitida de coliformes a $45^{\circ} \mathrm{C}$ em pescado refrigerado é de $10^{2}$ unidades formadoras de colônia por grama de alimento (UFC/g) sendo assim, nas amostras analisadas do produto de pargo, foram encontrados valores inferiores ao limite determinado pela legislação vigente, estando, portanto, de acordo com os padrões estabelecidos. Em dezembro de 2020, a legislação atual será substituída pela IN n. 60 (Brasil, 2019) onde a única diferença seria a substituição da contagem de coliformes a $45^{\circ} \mathrm{C}$ por análise de Escherichia coli para o produto desenvolvido. Estes resultados contrastam com os obtidos por Silva (2006), ao analisar hambúrguer de pescada amarela e de bagre rosado, onde detectou valores acima dos limites estabelecidos pela legislação, indicando falhas no procedimento de higiene e manipulação durante o processamento e conservação. Não foi detectada presença de Salmonella nas amostras analisadas, a presença da mesma normalmente está associada à contaminação durante a manipulação ou por contaminação do ambiente, sua presença constitui um grande problema para a saúde pública, devido ao seu caráter patogênico (Vaz, 2005). O resultado obtido para estafilococos coagulase positiva encontra-se dentro dos padrões da legislação vigente que permite no máximo $<5 \times 10^{2} \mathrm{UFC} / g$ para produtos a base de pescado refrigerado, incluindo embutido de peixe (Brasil, 2001). Galvão (2009) em estudo com salsicha a base de piramutaba (Brachyplatystama vaillanti) também obteve resultados dentro dos padrões da legislação para estafilococos coagulase positiva indicando boas condições de higiene no processamento e dos manipuladores. Assim como a salsicha tipo Frankfurter elaborada com bagre e cavalinha (Nkrumah; Akwetey, 2018). 
O gás sulfídrico $\left(\mathrm{H}_{2} \mathrm{~S}\right)$ se forma na carne através da hidrólise química, enzimática ou microbiana, sua detecção indica o grau de decomposição do pescado (Huss, 1999). A legislação brasileira considera deteriorado e, portanto, impróprio para o consumo, o pescado com reação positiva de gás sulfídrico (Brasil, 2017). O teor de histaminas também é um método proposto como critério de qualidade de pescado, explicado pelos baixos níveis detectados em peixe recém-capturado, aumentando com a sua deterioração (Souza et al., 2015). Para histaminas, a legislação brasileira considera um nível máximo de 100 ppm no músculo nas espécies pertencentes às famílias Scombridae, Scombresocidae, Clupeidae, Coryyphaenidae (Brasil, 1997). No estudo atual, o pargo, escolhido para o desenvolvimento do produto não está na lista das espécies susceptíveis preconizadas pela legislação brasileira, entretanto, devido aos riscos associados à captura e ao resfriamento inadequado nos barcos de pesca e a alta toxicidade da histamina, se fez necessário a investigação. Resultados diferentes do presente estudo foram encontrados. Em trabalho em que filés de peixes marinhos congelados foram analisados, Soares et al. (1998) detectaram teores de gás sulfídrico $\left(\mathrm{H}_{2} \mathrm{~S}\right)$ em $62 \%$ das amostras analisadas indicando estágio avançado de decomposição. Na mesma pesquisa, a histamina estava fora dos padrões da legislação em $37 \%$ das amostras analisadas.

Estudos com desenvolvimento de salsichas de outras espécies tem conseguido menor percentual de aceitação do que o presente trabalho. Oliveira Filho (2009) testando diferentes formulações de salsicha de tilápia, obteve a aceitabilidade global de $78,1 \%$ dos julgadores, semelhante a Gonçalves et al. (2009) que obtiveram aceitação global de $75,6 \%$ com salsicha de piramutaba contendo $30 \%$ de saborizante de camarão.

Em trabalho com elaboração de embutido cozido tipo salsicha com carne mecanicamente separada de resíduos de filetagem de tilápias do Nilo, Lago (2015) avaliou sensorialmente o produto utilizando uma escala de 9 pontos, obtendo a maioria dos resultados percentuais de aceitação dos atributos inferiores ao do presente estudo. Para os atributos odor, cor, e sabor os melhores resultados encontrados foram $75,0 \%, 53,12 \%$ e $75,0 \%$, respectivamente, enquanto que no trabalho atual para os mesmos atributos os percentuais foram $85,05 \%$; $66,66 \%$ e $85,05 \%$. Apenas para o atributo textura o resultado do trabalho com salsicha de tilápia foi superior apresentando $78,1 \%$ enquanto que a aceitação do presente estudo foi de $70,11 \%$. Tal resultado para a salsicha de pargo pode ser explicado analisando as observações apontadas nos atributos, que, para textura, foram registradas algumas opiniões: "achei meio seco", "secura", "esfarela bastante". Nos dois trabalhos, o atributo cor foi o de menor aceitação explicado pela aparência clara e não habitual das salsichas. $O$ atributo cor, no trabalho atual, recebeu alguns comentários, tais como: "muito pálido", "muito claro", "pálido".

Lago et al., (2017) desenvolvendo salsichas de tilápia formuladas com $50 \%$ de CMS encontrou entre as maiores médias o atributo sabor e a menor média para o atributo cor. Porém, a principal diferença encontrada entre os estudos está nas médias dos atributos textura, que no trabalho atual, obteve escores baixos diferindo do estudo da salsicha de tilápia que obteve a maior média atribuída a este atributo.

Estudo que desenvolveu salsichas de peixe ciprinídeo do Oriente, Capoeta umbla, encontrou boa aceitação nas amostra que usaram polifosfato de sódio com destaque para a categoria textura (Özpolat; Guran, 2017), diferente do presente estudo. Semelhantemente, Pourashouri et al. (2020) encontraram melhores resultados em aceitação usando emulsionantes em salsicha de carpa prateada. Enquanto Özpolat e Patir (2016) encontraram destaque no atributo odor em salsichas de peixes: boga Chondrostoma regium, bardo Luciobarbus mystaceus e ciprinídeos Capoeta trutta semelhantemente ao presente trabalho.

Os resultados de intenção de compra do presente trabalho mostraram-se inferiores a Lago (2015) que obteve como maior percentual em intenção de compra a segunda escala "provavelmente compraria"; na pesquisa atual, foi atribuída a maior porcentagem para talvez sim/talvez não $(32,1 \%)$.

\section{Conclusões}

O produto elaborado, salsicha de pargo, obteve condições higiênico-sanitárias satisfatórias, boa qualidade nutricional e alto índice de aceitação sensorial com destaque para os atributos avaliados odor e sabor e com um resultado de intenção de compra mediana. Assim, o produto mostrou-se ser uma alternativa viável e nutritiva para processamento industrial do pargo de tamanho reduzido proveniente da pesca de arrasto valorizando a matéria-prima podendo acrescentar valor de mercado, Pode ser uma alternativa na alimentação infantil tendo em vista as características do produto.

\section{Agradecimento}

Aos técnicos de laboratório que auxiliaram na pesquisa e à CAPES.

\section{Referências}

ALFARO, A.T.; LANES, G.F. C.; TORRES, L.M.; SOARES, G.J.D.; PRENTICE, C.H. Parâmetros de processamento e aceitabilidade de apresuntado elaborado com surimi de pescada-foguete (Macrodon ancylodon). Alimentos e Nutrição, v.15, n.3, p.259265, 2004.

AOAC. ASSOCIATION OF OFFICIAL ANALYTICAL CHEMISTS. Official Methods of Analysis 19 ed. Gaithersburgh, Maryland: Association of Official Analytical Chemists International 2007.
AZAMBUJA, H.G.P.; MARQUES, R.V.; PERIUS, D.B.; SANTO, M.L.P.E. Acompanhamento e avaliação tecnológica do enlatamento da anchoíta (Engraulis anchoita) em molho com tomate. VETOR-Revista de Ciências Exatas e Engenharias, v.24, n.1, p.21-32, 2016.

BADOLATO, E.S.; CARVALHO, J.B.D.; MELLO, M.R.P.D.A.; TAVARES, M.; CAMPOS, N.C.; AUED-PIMENTEL, S.; MORAIS, C.D. Composição centesimal de ácidos graxos e valor calórico de cinco espécies de peixes marinhos nas diferentes estações do ano. Revista Instituto Adolfo Lutz, v.54, n.1, p.27-35, 1994. 
BRASIL. Laboratório Nacional de Referência Animal/LANARA. Métodos Analíticos Oficiais para o controle de produtos de origem animal e seus ingredientes. Métodos físicos e químicos. Brasília, DF, 1981. v. 2. cap. 11. Pescado Fresco.

BRASIL. Ministério da Agricultura e do Abastecimento. Portaria $\mathrm{n}^{\circ}$ 185, de 13 de maio de 1997. Institui o Regulamento Técnico de Identidade e Qualidade do Peixe Fresco (Inteiro ou Eviscerado). Diário Oficial da União, Brasília, 19 de maio de 1997.

BRASIL. Ministério da Agricultura. Instrução Normativa n. 4, de 31 de março de 2000. Aprova os regulamentos técnicos de identidade e qualidade de carne mecanicamente separada, de mortadela, de lingüiça e de salsicha. Diário Oficial [da] República Federativa do Brasil, Brasília, 5 de abril de 2000. Seção 1, p. 6-10.

BRASIL. Ministério da Saúde. Agência Nacional de Vigilância Sanitária. Resolução - RDC n 12, de 02 de janeiro de 2001. Padrão microbiológico para alimentos. Disponível em:<http://portal.anvisa. gov.br/documents/33880/2568070/RDC_12_2001.pdf/15ffddf6-37674527-bfac-740a0400829b>. Acesso em: 06 dez. 2016.

BRASIL. Ministério da Agricultura, Pecuária e Abastecimento. Instrução Normativa 62, de 26 de agosto de 2003. Métodos analíticos oficiais para análises microbiológicas para o controle de produtos de origem animal e água. Diário Oficial [da] República Federativa do Brasil. Brasília, p 14-50. 18 de setembro de 2003. Seção I.

BRASIL. Ministério da Agricultura, Pecuária e Abastecimento - MAPA. DECRETO n 9.013, de 29 de março de 2017.

Regulamenta a lei $n^{\circ} 1.283$, de 18 de dezembro de 1950 , e a lei $n^{\circ} 7.889$, de 23 de novembro de 1989, que dispõem sobre a inspeção industrial e sanitária de produtos de origem animal. Diário Oficial da União, Brasília, 30 de março de 2017, n. 62, Seção 1: p. 3-26.

BRASIL. Ministério da Saúde. Agência Nacional de Vigilância Sanitária. Instrução Normativa n.60, de 23 de dezembro de 2019. Estabelece as listas de padrões microbiológicos para alimentos. Diário Oficial da União, Brasília, 26 de dezembro de 2016, n. 249 , Seção 1: p. 133.

CARMO, F.B.T.; MÁRSICO, E.T.; SÃO CLEMENTE, S.C.; CARMO, R.P.; FREITAS, M.Q. Histamina em conservas de sardinha. Ciência Animal Brasileira, v.11, n.1, p.174-180, 2010.

COLEMBERGUE, J.P.; GULARTE, M.A.; ESPIRITO SANTO, M.L.P. Caracterização química e aceitabilidade da sardinha (Sardinella brasiliensis) em conserva adicionada de molho com tomate. Alimentos e Nutrição, v.22, n.2, p.273-278, 2011.

COZER, N.; SIGNOR, A.; FEIDEN, A.; SILVA, A.M.; FEIDEN, A.; BOSCOLO, W.R. Enlatamento do jundiá: caracterização centesimal, microbiológica e sensorial do produto final. Boletim do Instituto de Pesca, v.1, n.40, p.61-68, 2014.

DUTCOSKY, S.D. Análise Sensorial de Alimentos. Curitiba: Editora Universitária Champagnat, 2011. 426p.

FAO. The Codex Alimentarius Commission and the FAO/ WHO Food Standards Programme. Special Publications. Food Labelling: complete texts. FAO, Roma, 2005. Disponível em: <http://www.codexalimentarius.net/web/publications_es.jsp>. Acesso em: 25 abr. 2017

FIPERJ - Fundação Instituto de Pesca do Estado do Rio de Janeiro. Boletim Estatístico da Pesca do Estado do Rio de Janeiro - Anos 2011 e 2012. Niterói: Fundação Instituto de Pesca do Estado do Rio de Janeiro, 2013. 93p. Disponível em:< file: http://www.fiperj.rj.gov.br/index.php/publicacao/index/1>. Acesso em: 27 de abr. de 2017.
GALVÃO, G.C.D.S. Influência dos Substitutos de Gordura na Salsicha de Pescado Elaborada com Resíduos da Filetagem da Piramutaba Brachyplatystama vaillanti (Valenciennes, 1840). 132f. Dissertação (Mestrado) - Instituto de Tecnologia, Universidade Federal do Pará, 2008. Disponível em:<http:// ppgcta.propesp.ufpa.br/ARQUIVOS/dissertacoes/2008/Giane\%20 Galv\%C3\%A3o.pdf>. Acesso em: 27 de abr. de 2017.

GLORIA, M.B.A.; SOARES, V.F.M. Comparison of fluorometric methods for the determination of histamine fish. Arquivo de Biologia e Tecnologia, v.36, n.2, p.229-235, 1993.

GONÇALVES, A.A. Formatados e Reestruturados (Hambúrguer, Nuggets etc.). In: GONÇALVES, A.A. Tecnologia do Pescado: Ciência, Tecnologia, Inovação e Legislação. São Paulo: Atheneu, 2011. cap. 01, p. 235-245.

GONÇALVES, A.A.; NOGUEIRA, W.M.; LOURENÇO, L.F.H. Aproveitamento do descarte do processamento da piramutaba (Brachyplatystoma vaillantii) e do camarão-rosa (Farfantepenaeus subtilis) na produção de salsicha sabor camarão. Boletim do Instituto de Pesca, v.35, n.4, p.623-635, 2009.

HAIMOVICI, M.; MARTINS, A. S.; FIGUEIREDO, J. L. D.; VIEIRA, P. J. C. Demersal bony fish of the outer shelf and upper slope of the southern Brazil Subtropical Convergence Ecosystem. Marine Ecology Progress Series, v.108, n.1, p.57-77, 1994.

HUSS, H.H. Garantia de qualidade dos produtos da pesca. Roma: FAO, 1997. Documento Técnico sobre as Pescas, 334. 176p. Disponível em: < http://www.fao.org/docrep/003/T1768P/ T1768P01.htm >. Acesso em 17 de mar. de 2017.

KELLEHER, K. Discards in the world's marine fisheries. An update. FAO Fisheries Technical Paper No 470. Rome, FAO, 2005. 131 pp. Includes a CD-ROM. Disponível em: <http://www. fao.org/docrep/008/y5936e/y5936e00.HTM >. Acesso em 15 de mar. de 2017

LAGO, A.M.T. Embutido tipo salsicha utilizando carne mecanicamente separada de tilápia: uma alternativa para o aproveitamento de resíduo da filetagem. $231 \mathrm{f}$. Dissertação (Mestrado) - Universidade Federal de Lavras, 2015. Disponível em: <http://repositorio.ufla.br/jspui/bitstream/1/10310/1/ DISSERTACAO_Embutido\%20tipo\%20salsicha\%20utilizando\%20 carne\%20mecanicamente.pdf>. Acesso em: 20 de abr. de 2017.

LAGO, A.M.T.; VIDAL, A.C.C.; SCHIASSI M.C.E.V.; REIS, T.; PIMENTA, C. PIMENTA, M.E.S.G Influence of the addition of minced fish on the preparation of fish sausage: effects on sensory properties. Journal of Food Science, v.82, n. 2, p. 492-499, 2017

LANFER-MARQUEZ, U.M.; MIRA, N.V.M. Avaliação da composição centesimal, aminoácidos e mercúrio contaminante de surimi. Ciência e Tecnologia de Alimentos, 25: 665-671, 2005.

LEE, C.M. Surimi process technology. Food Technology, 40(11): 69-80, 1984.

MELO, H.M.G.; MOREIRA, R.T.P.; DÁLMAS, S.; MACIEL, M.I.S.; BARBOSA, J.M.; MENDES, E.S. Viabilidade da utilização da carne mecanicamente separada (CMS) de tilápia do Nilo na elaboração de um produto tipo "mortadela". ARS Veterinária, v.27, p.22-29, 2011.

MARQUES, L.F.; NUNES, J.S.; CASTRO, D.S.; ARAUJO, L.K.; SALES, M.L.S. Avaliação da qualidade de linguiça de Tilápia do Nilo (Oreochromis niloticus). Revista Semiárido De Visu, v.2, n.1, p.202-209, 2012.

NKRUMAH, T.; AKWETEY, W.Y. Microbiological and Nutritional Properties of Frankfurter-Type Fish Sausage. International Journal of Nutrition, v.2, n.4, p.27-34, 2018. 
OLIVEIRA, E.C.M.; OLIVEIRA, E.R.; LIMA, L.C.O.; BOAS, E.V.B.V. Composição centesimal do cogumelo do sol (Agaricus blazei). Revista Universidade Alfenas, v.5, p.169-172, 1999.

OLIVEIRA FILHO, P.R. Elaboração de embutido cozido tipo salsicha com carne mecanicamente separada de resíduos de filetagem de tilápias do Nilo. 115f. Tese (Doutorado) - Centro de Aquicultura, Universidade Estadual Paulista, 2009. Disponível em: <https://repositorio.unesp.br/bitstream/handle/11449/100241/ oliveirafilho_prc_dr_jabo.pdf?sequence=1>. Acesso em: $18 \mathrm{de}$ mar. de 2017.

ÖZPOLAT1, E.; PATIR, B. Determination of shelf life for sausages produced From some freshwater fish using two different Smoking methods. Journal of Food Safety, v.3, n.6, p.69-76, 2016.

ÖZPOLAT E.; GURAN, H.S. Combined effect of sodium polyphosphate and smoking on quality parameters of fish (Capoeta umbla) sausage. Iranian Journal of Fisheries Sciences, v.16, n.1, p. 86- 95, 2017.

PEIXOTO, M.R.S.; SOUSA, C.L.; MOTA, E.S. Utilização de pescada (Macrodom ancylodon) de baixo valor comercial na obtenção de surimi para elaboração de moldado sabor camarão. Boletim do Centro de Pesquisa de Processamento de Alimentos, v.18, n.2, p. 151-162, 2000

PIRES, D.R.; SILVA, P.P.O.; AMORIM, E.; OLIVEIRA, G.M. Espécies de pescado subexplotadas e seu potencial para elaboração de subprodutos com valor agregado. Revista Verde de Agroecologia e Desenvolvimento Sustentável, v.8, n.5, p.148157, 2014.

PIZATO, S.; KRAIESKI, J.; SARMENTO, C.; PRENTICE, C. Avaliação da qualidade tecnológica apresentada por tilápia do Nilo (Oreochromis niloticus) enlatada. Semina: Ciências Agrárias, v.33, n.2, p.667-674, 2012.

POURASHOURI, P.; SHABANPOUR B.; KORDJAZI, M.; JAMSHIDI, A. Characteristic and shelf life of fish sausage: fortification with fish oil through emulsion and gelled emulsion incorporated with green tea extract. Journal of the Science of Food and Agriculture, v.100, n.12, p.1-26, 2020.
QUIRINO-DUARTE, G.; SOUZA, M.D.; CARNEIRO, M.H.; SERVO, G.D.M. Composição quali-quantitativa da categoria "mistura" na pesca de arrasto duplo de portas médio desembarcada nos municípios de santos e Guarujá, São Paulo, Brasil. Boletim do Instituto de Pesca, v.35, n.3, p.461-474, 2009.

SILVA, J.J.; CHAMUL, R.S. Composition of marine and freshwater finfish and shellfish species and their products. In: MARTIN, R.E.; PAINE, E.; FLICK, E.J.; DAVIS L.M. (Eds.). Marine and freshwater products handbook. Technomic Publishing Company, 2000. p. 31- 46. Disponível em : < http://www.scirp.org/ (S(351jmbntvnsjt1aadkposzje))/reference/ReferencesPapers. aspx?ReferencelD=1115925 >. Acesso em: 19 de mar. de 2017.

SOUZA, A.L.M.; CALIXTO, F.A.A.; MESQUITA, E.F.M.; PACKNESS, M.P.; AZEREDO, D.P. Histamina e rastreamento de pescado: revisão de literatura. Arquivos do Instituto Biológico, v.82, p. 01-11, 2015.

TENUTA-FILHO, A.; JESUS, R.S. Aspectos da utilização de carne mecanicamente separada de pescado como matériaprima industrial. Boletim da Sociedade Brasileira de Ciência e Tecnologia de Alimentos, v.37, n.2, p.59-64, 2003.

VAZ, S.K. Elaboração e caracterização de linguiça fresca "tipo toscana" de tilápia (Oreochromis niloticus). 113f. Dissertação (Mestrado) - Universidade Federal do Paraná, 2005. Disponível em: <http://www.acervodigital.ufpr.br/bitstream/handle/1884/2169/ Disserta\%E7ao\%20simone\%20vaz.pdf?sequence=1>. Acesso em: 13 de mar. de 2017.

VIANNA, M.; KEUNECKE, K.A.; MATOS, F.J.P.; ALMEIDA, H.L.; KASSUGA, A.D.; ARANTES, C.C. Caracterização instantânea das consequências da pesca com utilização de redes de arrasto dentro da Baía de Guanabara, Rio de Janeiro. In: CONGRESSO BRASILEIRO DE DEFESA DO MEIO AMBIENTE, 7, Rio de Janeiro, 28 p., agosto/2003. Anais... Rio de Janeiro: Universidade Federal do Rio de Janeiro. 1 CD-ROM. 